\title{
Face Trees for Expression Recognition
}

\author{
Mojtaba Kolahdouzi, Alireza Sepas-Moghaddam, and Ali Etemad \\ Dept. ECE and Ingenuity Labs Research Institute, Queen's University, Kingston, Canada
}

\begin{abstract}
We propose an end-to-end architecture for facial expression recognition. Our model learns an optimal tree topology for facial landmarks, whose traversal generates a sequence from which we obtain an embedding to feed a sequential learner. The proposed architecture incorporates two main streams, one focusing on landmark positions to learn the structure of the face, while the other focuses on patches around the landmarks to learn texture information. Each stream is followed by an attention mechanism and the outputs are fed to a two-stream fusion component to perform the final classification. We conduct extensive experiments on two largescale publicly available facial expression datasets, AffectNet and FER2013, to evaluate the efficacy of our approach. Our method outperforms other solutions in the area and sets new state-ofthe-art expression recognition rates on these datasets.
\end{abstract}

\section{INTRODUCTION}

Facial expressions have been regarded as one of the most common non-verbal cues for conveying human emotions [20], [34]. Therefore, automatic facial expression recognition (FER) from images has received considerable attention in recent years [30], [29], [28]. Naturally, deep learning methods have recently become the dominant approach for FER, starting with early work using a shallow CNN and a support vector machine (SVM) in [32]. More sophisticated deep learning solutions have recently been proposed. In [4], a novel attention method which causes the method to focus on moving facial muscles was proposed. In [35] another attention-based method was proposed to rank the importance of facial regions. To tackle data imbalance, which is a common issue in FER, a novel loss function was proposed in [14]. In [17], a novel data augmentation approach for FER was proposed. By altering the standard skip connections in a ResNet model with a differentiable function, the Bounded Residual Gradient Network (BReGNet) was proposed in [10]. By adding trainable parameters to BReG-Net, the same authors later proposed BReG-NeXt [9], which offers state-of-the-art results on AffectNet [26] and FER2013 [7] datasets. More recently, self-supervised and contrastive approaches have also begun to gain popularity in this area, but have in many cases used either videos [28] or multi-view [31] data as opposed to the work presented in this study which focuses on a single image.

In order to make use of the existing relationships between facial regions, exploiting graph topologies in neural networks

This work was funded by Irdeto Canada Corporation and the Natural Sciences and Engineering Research Council of Canada (NSERC).

(C) 2021 IEEE. Personal use of this material is permitted. Permission from IEEE must be obtained for all other uses, in any current or future media, including reprinting/republishing this material for advertising or promotional purposes, creating new collective works, for resale or redistribution to servers or lists, or reuse of any copyrighted component of this work in other works

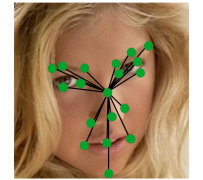

(a) $52.41 \%$

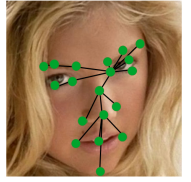

(b) $54.47 \%$

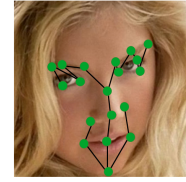

(c) $56.1 \%$



(d) $55.42 \%$
Fig. 1: A face image along with 4 random trees. Corresponding recognition rates are written below each image.

for FER has gained popularity [36], [42], [22]. In [41], Gabor features were combined and used to feed a gated recurrent unit. In [42], the relationship between facial landmarks were provided by using their psychological semantic relationships. The resulting topology was then processed by a graph network. Despite clear effectiveness of such graph-based methods, there is no unique consensus on the topology of the underlying graphs that should be used for FER. Current graph-based FER methods either construct complete graphs [41], [37], [19] or use pre-defined topologies [42], [38], [36], both of which do not capture the optimal connections. The topologies of these graphs can have considerable effects on the performance of networks, including sequential learners. Figure 1 shows a face image along with 4 random trees that were built on the extracted facial landmarks. Traversing these trees results in 4 different sequence-based embeddings that can be fed to 4 independent LSTM networks for a 7-class FER task. The corresponding recognition rates are shown below each image in the figure where we see a considerable range given the different topologies.

In this paper, we propose an end-to-end deep FER architecture to learn an optimal graph of the face in the form of a tree topology whose traversal creates a sequence, which is used to form the input embedding for a sequence learner. To this end, we consider a weighted complete graph, from which we generate a minimum-cost spanning tree and choose the optimal edges. Our end-to-end model consists of three blocks. In the first block, we perform the tree topology learning. Our optimized tree is then used in a two-stream framework, structure and texture. In the structure stream, we traverse the optimized tree to form a sequence, whose resultant embedding is then fed to an LSTM. This is followed by a soft attention mechanism. In the texture stream, we encode the local patches around the extracted facial landmarks, prior to learning a separate LSTM network. The final embedding is fused with that of the structure stream. To the best of our knowledge, this paper is the first that optimizes the order of relevant facial regions prior to recurrently learning their dependencies for FER. Our experiments on two large-scale 
public FER datasets, AffectNet [26] and FER2013 [7], set new state-of-the-art results.

In summary, our main contributions are as follows. (1) We propose a novel end-to-end architecture to utilize optimal face tree topologies. (2) Our experiments show that the two-stream solution has a positive impact on the results. Moreover, we observe that given our tree topology optimization, a different face tree is learned for each dataset. Lastly, our solution sets new state-of-the-art values on large-scale AffectNet [26] and FER2013 [7] datasets.

\section{Proposed Method}

\section{A. Problem and Solution Overview}

Graphs can model facial components [42], [2] in which vertices denote facial landmarks while edges correspond to the relationship between these landmarks [15]. As evident from Figure 1, the topology of such graphs can affect the performance of subsequent models that use it. Nevertheless, no prior work has been proposed to optimally learn the topology of facial graphs. To address this gap, we propose a method to learn the optimum tree capable of characterizing these relationships. Next, in order to learn critical information from the face, we consider two key streams, structure and texture. The former learns the structure while the latter learns the texture of key patches in the face, both of which consider the optimized tree. Our method traverses the optimized tree to form a sequence, which is then learned recurrently using a sequential learner, in this case an LSTM. Figure 2 illustrates the architecture of our proposed pipeline.

\section{B. Tree Topology Learning}

We intend to first learn an optimal face tree topology to be used in subsequent components. To this end, we first detect the facial landmarks from the input images using a deep regression architecture [24]. Next, we construct a weighted fully connected graph, $k_{n}$, from the landmarks. We then find the optimum minimum-cost spanning tree considering the weights of $k_{n}$, whose traversal generates a sequence by which we form an embedding to feed to a sequential learner. We use the Prim's algorithm [13] to solve the problem of finding a minimum-cost spanning tree. The built tree is then traversed using Preorder depth-first order [18], with the starting point being the most centered landmark, i.e., nose tip. During the traversal, when a leaf node is reached, the traversal backtracks to go back to the starting point. Figure 3 depicts the tree traversal process.

Our model then updates the learnable weights associated with $k_{n}$. To this end, we adopt a metaheuristic optimization algorithm as used in [21]. For the optimizer's objective function we use the cost function as follows:

$$
\begin{array}{r}
J(w)=\frac{1}{3 m}\left(\sum_{i=1}^{m} L_{1}^{f o c}\left(\hat{y}^{(i)}, y^{i}\right)+\right. \\
\sum_{i=1}^{m} L_{2}^{f o c}\left(\hat{y}^{(i)}, y^{i}\right)+ \\
\left.\sum_{i=1}^{m} L_{3}^{f o c}\left(\hat{y}^{(i)}, y^{i}\right)\right)
\end{array}
$$

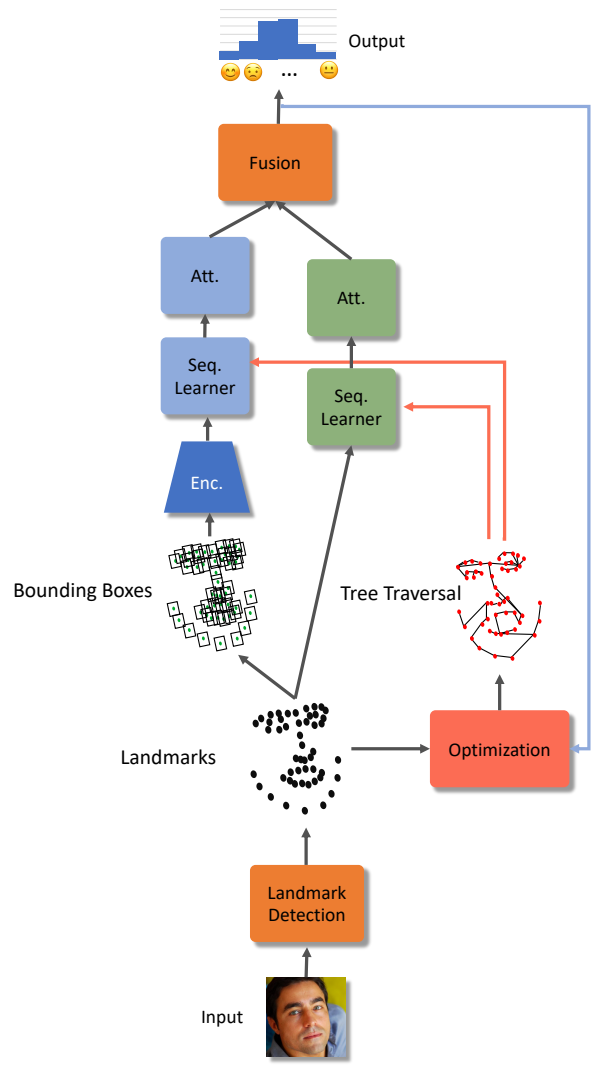

Fig. 2: Our proposed pipeline.

where $m$ is the size of the training set, $L_{1}^{f o c}(x)$ is the focal loss function selected to maximize performance for the fusion step, and $L_{2}^{f o c}(x)$ and $L_{3}^{f o c}(x)$ are respectively the focal loss functions for structure and texture streams. We use focal losses since the data distribution in most FER datasets is highly skewed [23]. Here, $L^{f o c}$ is defined as:

$$
L^{f o c}\left(p_{b}\right)=-\alpha_{b}\left(1-p_{b}\right)^{\gamma} \log \left(p_{b}\right),
$$

where $\gamma$ is the focusing parameter and $p_{b}$ is the binomial distribution, in which $\hat{y}$ is the probability of $y$ being 1 and $1-\hat{y}$ is the probability of $y$ being 0 . We set $\gamma=2$ and $\alpha_{b}=0.25$ as recommended in [23].

At each epoch, the metaheuristic algorithm generates a set of weights associated with $k_{n}$, from which we generate the minimum-cost spanning tree, whose traversal results in a sequence. Next, this sequence is frozen and the subsequent structure and texture streams are fully trained. The reason for converting the obtained graph to a tree is that the acyclic nature of trees is better suited for traversal, hence sequence generation.

\section{Structure Stream}

The coordinates of the facial landmarks which were extracted in the tree topology learning step, along with the obtained sequence, are the inputs to the structure stream (see Figure 2). By using this sequence, we generate an embedding to be fed to an LSTM network with peephole connections [6]. 


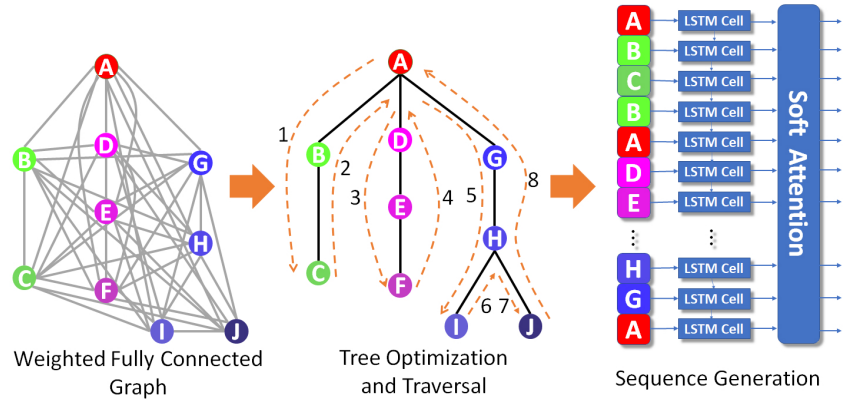

Fig. 3: Details of the face tree topology learning. Traversing the tree results in A-B-C-B-A-D-E-F-E-D-A-G-H-I-H-J-H$\mathrm{G}-\mathrm{A}$, which is then fed to an LSTM network.

Following the LSTM network, we add an attention step [27] to better focus on important parts. To this end, the hidden state vectors $h_{i}$ of the LSTM cells are multiplied by a set of coefficients $\alpha_{i}$ to determine the level of attention:

$$
\alpha_{i}=\frac{e^{u_{i}}}{\sum_{k=1}^{n} e^{u_{k}}},
$$

where, $n$ is the number of LSTM units and $u_{i}$ is calculated as:

$$
u_{i}=\tanh \left(W_{h} h_{i}+b_{h}\right) .
$$

In Eq. $4, W_{h}$ and $b_{h}$ are the trainable weights and biases. The attentive output is calculated $H=\sum_{i} \alpha_{i} h_{i}$, where $H$ is the final embedding of the structure stream.

\section{Texture Stream}

Similar to the structure stream, the inputs to the texture stream are the facial landmarks and the sequence generated in the face tree topology learning step. However, unlike the structure stream, we focus on the texture of the facial images. To this end, we first form $n \times n$ patches centered around each facial landmark. We then crop these patches from the images and feed them to a an encoder (ResNet50) pre-trained on the VGG-Face2 dataset [3]. Through empirical experimentation, we find the patch size of $17 \times 17$ pixels to yield the best results. We stack these embeddings in accordance to the sequence obtained from the tree topology learning step (see Figure 3). The resulting embedding is then passed onto an LSTM network followed by a soft attention mechanism.

\section{E. Fusion}

In order to fuse the outputs of the structure and texture streams, we adopt the two-stream fusion strategy used in [8], [40]. This fusion strategy has yielded state-of-the-art results in a number of areas [8]. Here, we use two dense layers as encoders for each of the input streams, and generate streamspecific features. Then, soft attention is adopted to learn the weight $\eta$ by using $\eta=\operatorname{softmax}\left(\tanh \left(W_{f}\left[T^{*}, S^{*}\right]+b_{f}\right)\right)$, where $W_{f}$ and $b_{f}$ are trainable weights and biases, respectively. $T^{*}$ and $S^{*}$ are the stream-specific features for texture and structure. Next, a dense layer is utilized to learn the optimal association across the embedding-specific features.

\section{EXPERIMENTS}

Implementation. The entire architecture is implemented using TensorFlow [1] and is trained using a pair of Nvidia RTX 2080 Ti GPUs. For optimizing the weights of the model, we adopt ADAM optimizer [16] with the learning rate, first-momentum decay, and second-momentum decay of $0.001,0.9$, and 0.99 , respectively.

Datasets. We test our model on two large in-the-wild datasets: (i) AffectNet, comprising more than 1 million images with 8 different expression categories, and (ii) FER2013, which includes 33000 images and has 6 expression categories.

\section{A. Performance}

The recognition rates (RR) obtained by our model and other state-of-the-art benchmarking methods are presented in Table I. For AffectNet dataset, our model shows an improvement of $1.52 \%$ and $3.28 \%$ over the BreG-NeXt50 and BreG-NeXt32, the current state-of-the-art FER methods. For FER2013 dataset, our proposed pipeline outperforms the current state-of-the-art FER method, i.e., BreG-Next50, showing a performance improvement of $1.13 \%$.

For a head-to-head comparison between our proposed model and BreG-NeXt50 (best performing benchmark), we present F1 scores for each expression by using a one-vs-all scheme [5] in Figure 4. Concerning AffectNet, our model exhibits higher F1 scores than BreG-NeXt50 in all the 8 expression classes except happy and neutral. AffectNet is skewed toward happy and neutral expressions. Despite this imbalance, our pipeline shows better results in the minority expression classes which are more difficult to recognize. With regards to FER2013, our pipeline achieves better F1 scores in 4 out of the 7 expressions. The exceptions are surprise, happy, and neutral expressions, in which BreGNeXt50 performs slightly better.

Figure 5 depicts the confusion matrices obtained by our method. For AffectNet, the largest confusions occur for contempt-happy (where contempt is the true class and happy is the predicted class), sad-surprise, and neutral-happy cases. Confusing contempt with happy is common in FER systems, since it is very hard to distinguish between these two even for humans [9]. In terms of FER2013, the largest confusion occurs in the disgust-happy case. The reason is that disgust and happy are the minority and majority classes respectively. Although we use focal loss to tackle imbalanced data, some of the minority expressions were still classified as the majority classes. Other than disgust, our pipeline shows good performance in recognizing true classes in FER2013 dataset.

\section{B. Tree Topology Learning}

The topology evolution of the face trees during can provide insight into the learning process. Figure 6 illustrates the convergence of the minimum-cost spanning trees obtained by our method, for AffetNet and FER2013 datasets in iterations $1,10,30$, and 40 . In the first iteration, a random tree is generated by assigning random weights to the edges of the $k_{50}$. We observe that as training is being performed, face 
TABLE I: Evaluation metrics on AffectNet and FER2013.

\begin{tabular}{l|l|l|l}
\hline Dataset & Authors & Method & RR \\
\hline & Zeng et al. [39] & CNN & 57.31 \\
& Hewitt et al. [11] & CNN & 58 \\
& Hua et al. [12] & Ensemble & 62.11 \\
AffectNet & Chen et al. [4] & Facial mask & 61.50 \\
& Kollias et al. [17] & Augmentation & 60 \\
& Hasani et al. [9] & BreG-NeXt32 & 66.74 \\
& Hasani et al. [9] & BreG-NeXt50 & 68.50 \\
& Ours & Tree learning & $\mathbf{7 0 . 0 2}$ \\
\hline \multirow{5}{*}{ FER2013 } & Tang et al. [32] & CNN & 69.3 \\
& Mollahosseini et al. [25] & CNN & 66.4 \\
& Vielzeuf et al. [33] & CNN & 71.2 \\
& Hasani et al. [9] & BreG-NeXt32 & 69.11 \\
& Hasani et al. [9] & BreG-NeXt50 & 71.53 \\
& Ours & Tree learning & $\mathbf{7 2 . 6 6}$ \\
\hline
\end{tabular}
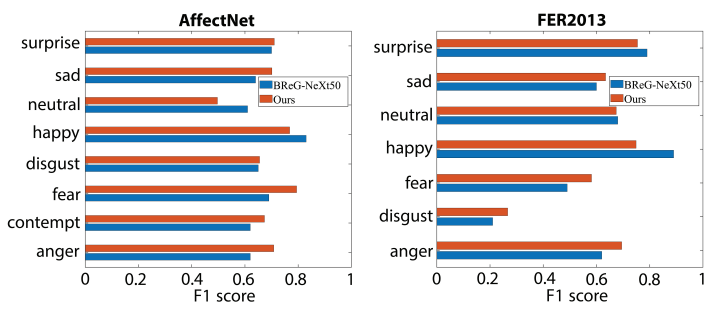

Fig. 4: Comparison of the obtained F1 scores.

TABLE II: Ablation experiments.

\begin{tabular}{c|c|c|c|c}
\hline \multirow{2}{*}{ Removed } & \multicolumn{2}{|c|}{ RR } & \multicolumn{2}{c}{ Drop in RR } \\
\cline { 2 - 5 } & AffectNet & FER2013 & AffectNet & FER2013 \\
\hline \hline Tree topology & $66.51 \%$ & $67.11 \%$ & $5.0 \%$ & $7.60 \%$ \\
Structure stream & $64.90 \%$ & $66.02 \%$ & $7.30 \%$ & $9.14 \%$ \\
Texture stream & $62.14 \%$ & $63.17 \%$ & $11.25 \%$ & $13.06 \%$ \\
Fusion strategy & $66.87 \%$ & $68.24 \%$ & $4.50 \%$ & $6.08 \%$ \\
\hline
\end{tabular}

trees tend to resemble the structure of human faces more and more. As an obvious example, face trees formed in iteration 40 for both AffectNet and FER2013 dataset closely resemble human faces around the jaw line, mouth, nose, and eyes, while still being customized for each dataset. Furthermore, in iteration 40, large variations between the two face trees of AffectNet and FER2013 occurs near the mouth and eye regions. This is not surprising, since these regions are highly informative in FER, and therefore these regions are customized for each dataset.

\section{Ablation Study}

To illustrate the contribution of each component of the proposed pipeline, we perform ablation experiments by systematically removing individual components of the model. Table II compares the recognition rates obtained by the following models: $(a)$ the model without tree topology learning where we adopt a random tree to form the input embeddings; (b) the model without the structure stream, in which the coordinates of facial landmarks along with the learned tree topology are utilized to form the embeddings; $(c)$ the model without structure stream where the output embedding of ResNet50 along with the learned tree topology is used to form the embeddings; and $(d)$ the model with a simple concatenation of the output embeddings from the structure and

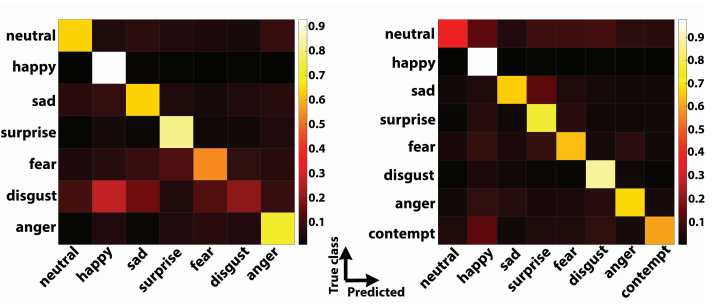

Fig. 5: Confusion matrices obtained by our model.

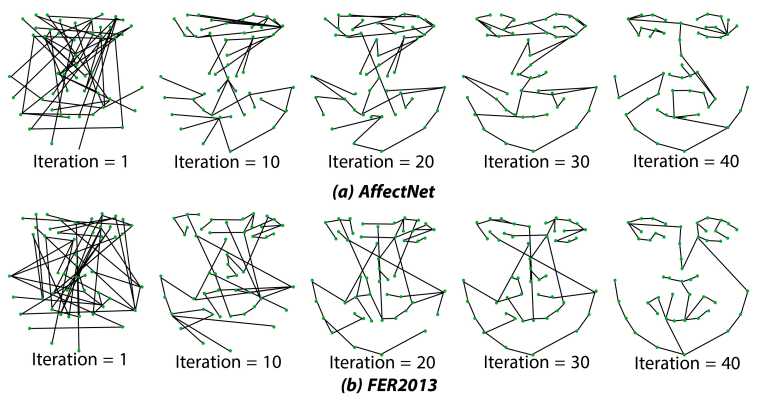

Fig. 6: Evolution of face trees during the training phase.

texture streams, instead of using the 2-stream fusion strategy. The results presented in Table II reveal that: (1) tree topology learning is an effective component in our proposed pipeline whose removal results in significant performance drops of $5.0 \%$ and $7.60 \%$, respectively for AffectNet and FER2013 datasets; (2) the contribution of the texture stream is higher than that of the structure stream due to the exploitation of the pre-trained ResNet50 in the texture stream; (3) relative contribution of the employed fusion strategy is lower than that of the tree topology learning. This demonstrates the importance of the tree topology learning in the pipeline.

\section{CONCLUSION}

In this paper we propose a novel end-to-end method called deep face topology network for facial expression recognition. Our model assigns weights to the edges of a complete graph representing all the possible connections between extracted facial landmarks and integrates them into an optimization process. These weights along with the complete graph are used to generate a minimum cost spanning tree (MST), whose traversal generates an optimal sequence. This sequence is used to form an embedding to feed an LSTM network. Our solution has been integrated in an end-toend two-stream pipeline. The first stream uses the spatial information of facial landmarks, while the other uses the texture information of patches around the landmarks. A fusion strategy has finally been adopted to combine the two. Experiments have been performed with two large-scale AffectNet and FER2013 datasets, where the results show the superiority of our method in comparison to prior work. The main limitation of this work is the optimization speed, which we will address in future works by using policy gradient methods and applying faster algorithms for finding the MST. 


\section{REFERENCES}

[1] M. Abadi, P. Barham, J. Chen, Z. Chen, A. Davis, J. Dean, M. Devin, S. Ghemawat, G. Irving, M. Isard, et al. Tensorflow: A system for large-scale machine learning. In 12th Symposium on Operating Systems Design and Implementation, pages 265-283, 2016.

[2] S. A. Angadi and S. M. Hatture. Face recognition through symbolic modeling of face graphs and texture. International Journal of Pattern Recognition and Artificial Intelligence, 33(12):1956008, 2019.

[3] Q. Cao, L. Shen, W. Xie, O. M. Parkhi, and A. Zisserman. Vggface2: A dataset for recognising faces across pose and age. In 13th IEEE International Conference on Automatic Face \& Gesture Recognition, pages 67-74, 2018.

[4] Y. Chen, J. Wang, S. Chen, Z. Shi, and J. Cai. Facial motion prior networks for facial expression recognition. In IEEE Visual Communications and Image Processing, 2019.

[5] M. Galar, A. Fernández, E. Barrenechea, H. Bustince, and F. Herrera. An overview of ensemble methods for binary classifiers in multi-class problems: Experimental study on one-vs-one and one-vs-all schemes. Pattern Recognition, 44(8):1761-1776, 2011.

[6] F. A. Gers and J. Schmidhuber. Recurrent nets that time and count. In IEEE-INNS-ENNS International Joint Conference on Neural Networks. Neural Computing: New Challenges and Perspectives for the New Millennium, volume 3, pages 189-194, 2000

[7] I. J. Goodfellow, D. Erhan, P. L. Carrier, A. Courville, M. Mirza, B. Hamner, W. Cukierski, Y. Tang, D. Thaler, D.-H. Lee, et al. Challenges in representation learning: A report on three machine learning contests. In International Conference on Neural Information Processing, pages 117-124, 2013.

[8] Y. Gu, K. Yang, S. Fu, S. Chen, X. Li, and I. Marsic. Hybrid attention based multimodal network for spoken language classification. In Association for Computational Linguistics. Meeting, volume 2018 , page 2379, 2018.

[9] B. Hasani, P. S. Negi, and M. Mahoor. Breg-next: facial affect computing using adaptive residual networks with bounded gradient. IEEE Transactions on Affective Computing, 2020.

[10] B. Hasani, P. S. Negi, and M. H. Mahoor. Bounded residual gradient networks (breg-net) for facial affect computing. In IEEE International Conference on Automatic Face \& Gesture Recognition, pages 1-7. IEEE, 2019.

[11] C. Hewitt and H. Gunes. Cnn-based facial affect analysis on mobile devices. arXiv preprint arXiv:1807.08775, 2018.

[12] W. Hua, F. Dai, L. Huang, J. Xiong, and G. Gui. Hero: Human emotions recognition for realizing intelligent internet of things. IEEE Access, 7:24321-24332, 2019.

[13] B. Jiang and L. Zhang. Research on minimum spanning tree based on prim algorithm. Computer Engineering and Design, 13, 2009.

[14] P. Jiang, G. Liu, Q. Wang, and J. Wu. Accurate and reliable facial expression recognition using advanced softmax loss with fixed weights. IEEE Signal Processing Letters, 27:725-729, 2020

[15] R. Jiang, A. T. Ho, I. Cheheb, N. Al-Maadeed, S. Al-Maadeed, and A. Bouridane. Emotion recognition from scrambled facial images via many graph embedding. Pattern Recognition, 67:245-251, 2017.

[16] D. P. Kingma and J. Ba. Adam: a method for stochastic optimization. arxiv. arXiv preprint arXiv:1412.6980, 22, 2014.

[17] D. Kollias, S. Cheng, E. Ververas, I. Kotsia, and S. Zafeiriou. Deep neural network augmentation: Generating faces for affect analysis. International Journal of Computer Vision, pages 1-30, 2020.

[18] D. C. Kozen. Depth-first and breadth-first search. In The Design and Analysis of Algorithms, pages 19-24. Springer, 1992.

[19] L. Lei, J. Li, T. Chen, and S. Li. A novel graph-ten with a graph structured representation for micro-expression recognition. In $A C M$ International Conference on Multimedia, pages 2237-2245, 2020.

[20] S. Li and W. Deng. Deep facial expression recognition: A survey. IEEE Transactions on Affective Computing, 2020.

[21] X. Li and X. Yao. Cooperatively coevolving particle swarms for large scale optimization. IEEE Transactions on Evolutionary Computation, 16(2):210-224, 2011.

[22] Y. Li, D. Tarlow, M. Brockschmidt, and R. Zemel. Gated graph sequence neural networks. arXiv preprint arXiv:1511.05493, 2015.

[23] T.-Y. Lin, P. Goyal, R. Girshick, K. He, and P. Dollár. Focal loss for dense object detection. In IEEE International Conference on Computer Vision, pages 2980-2988, 2017.

[24] J. Lv, X. Shao, J. Xing, C. Cheng, and X. Zhou. A deep regression architecture with two-stage re-initialization for high performance facial landmark detection. In IEEE Conference on Computer Vision and Pattern Recognition, pages 3317-3326, 2017.

[25] A. Mollahosseini, D. Chan, and M. H. Mahoor. Going deeper in facial expression recognition using deep neural networks. In IEEE Winter Conference on Applications of Computer Vision, pages 1-10, 2016.

26] A. Mollahosseini, B. Hasani, and M. H. Mahoor. Affectnet: A database for facial expression, valence, and arousal computing in the wild. IEEE Transactions on Affective Computing, 10(1):18-31, 2017.

[27] T. Rocktäschel, E. Grefenstette, K. M. Hermann, T. Kočiskỳ, and P. Blunsom. Reasoning about entailment with neural attention. arXiv preprint arXiv:1509.06664, 2015.

[28] S. Roy and A. Etemad. Spatiotemporal contrastive learning of facia expressions in videos. arXiv preprint arXiv:2108.03064, 2021.

[29] A. Sepas-Moghaddam, A. Etemad, F. Pereira, and P. L. Correia. Facial emotion recognition using light field images with deep attention-based bidirectional 1stm. In IEEE International Conference on Acoustics, Speech and Signal Processing, pages 3367-3371, 2020.

[30] A. Sepas-Moghaddam, A. Etemad, F. Pereira, and P. L. Correia. Capsfield: light field-based face and expression recognition in the wild using capsule routing. arXiv preprint arXiv:2101.03503, 2021.

[31] R. Shuvendu and A. Etemad. Self-supervised contrastive learning of multi-view facial expressions. In International Conference on Multimodal Interaction, 2021.

[32] Y. Tang. Deep learning using linear support vector machines. arXiv preprint arXiv: $1306.0239,2013$

[33] V. Vielzeuf, S. Pateux, and F. Jurie. Temporal multimodal fusion for video emotion classification in the wild. In ACM International Conference on Multimodal Interaction, pages 569-576, 2017.

[34] K. Wang, X. Peng, J. Yang, S. Lu, and Y. Qiao. Suppressing uncertainties for large-scale facial expression recognition. In IEEE/CVF Conference on Computer Vision and Pattern Recognition, pages 68976906, 2020.

[35] K. Wang, X. Peng, J. Yang, D. Meng, and Y. Qiao. Region attention networks for pose and occlusion robust facial expression recognition. IEEE Transactions on Image Processing, 29:4057-4069, 2020.

[36] H.-X. Xie, L. Lo, H.-H. Shuai, and W.-H. Cheng. Au-assisted graph attention convolutional network for micro-expression recognition. In ACM International Conference on Multimedia, pages 2871-2880, 2020.

[37] Y. Xie, T. Chen, T. Pu, H. Wu, and L. Lin. Adversarial graph representation adaptation for cross-domain facial expression recognition. In ACM International Conference on Multimedia, pages 1255-1264, 2020.

[38] X. Xu, Z. Ruan, and L. Yang. Facial expression recognition based on graph neural network. In International Conference on Image, Vision and Computing, pages 211-214, 2020.

[39] J. Zeng, S. Shan, and X. Chen. Facial expression recognition with inconsistently annotated datasets. In European Conference on Computer Vision (ECCV), pages 222-237, 2018.

[40] G. Zhang and A. Etemad. Rfnet: riemannian fusion network for eegbased brain-computer interfaces. arXiv preprint arXiv:2008.08633, 2020

[41] L. Zhong, C. Bai, J. Li, T. Chen, S. Li, and Y. Liu. A graphstructured representation with brnn for static-based facial expression recognition. In IEEE International Conference on Automatic Face \& Gesture Recognition, pages 1-5, 2019.

[42] J. Zhou, X. Zhang, Y. Liu, and X. Lan. Facial expression recognition using spatial-temporal semantic graph network. In IEEE International Conference on Image Processing (ICIP), pages 1961-1965, 2020. 